\title{
International production, structural change and public policies in times of pandemics
}

\author{
Andrea Coveri ${ }^{1} \cdot$ Dario Guarascio $^{2} \cdot$ Michael Landesmann $^{3}$
}

Published online: 22 July 2020

(c) Associazione Amici di Economia e Politica Industriale 2020

\section{JEL Classification $\mathrm{E} 10 \cdot \mathrm{E} 60 \cdot \mathrm{F} 40$}

The COVID-19 pandemic is reshaping the world economy at a pace never seen before. Both supply- and demand-side conditions have been affected, putting all relevant dimensions of the economy under unprecedent stress.

At the time of writing this introduction, the virus is continuing to infect and kill large numbers of people in several countries, especially in the US, Brazil and India. In Europe, the contagion curve has flattened almost everywhere through the widespread application of draconian lockdown and social distancing measures. By drastically reducing interpersonal contacts, such measures were instrumental to mitigate the health consequences of the pandemic. At the same time, they caused a dramatic drop in global GDP and employment, resulting in a major economic slowdown which is paving the way for a recession even worse than the one triggered by the Great Financial Crisis of 2008.

On the one hand, the economic meltdown requires urgently extraordinary anticrisis and pro-recovery policy efforts. On the other hand, the COVID-19 pandemic is likely to have structural and permanent effects on key variables as consumers' spending patterns and savings behavior, companies' propensity to invest, banks' willingness to provide loans, as well as on the organization of production on a global scale. Not less relevant, the fear of a new surge of contagion makes the economic environment radically uncertain with negative effects on national and international movements of people, goods and capital.

The COVID-19 pandemic, however, did not come 'out of the blue'. Both the emergence of COVID-19 as a threat to human beings as well as its fast diffusion on a global scale were linked to how global production and consumption became organized over the past decades. Analogously, the magnitude of the pandemic's

Dario Guarascio

dario.guarascio@uniroma1.its

1 University of Urbino "Carlo Bo", Urbino, Italy

2 Sapienza University of Rome, Rome, Italy

3 Vienna Institute for International Economic Studies (wiiw), Wien, Austria 
socio-economic consequences and their uneven impact across geographical areas, sectors, companies and social groups are, in many cases, the result of features that characterized the functioning of the world economy. The unprecedent character of this pandemic, moreover, has highlighted the differences in the capacities of local, national and supranational governments to cope with an emergency of this size. This refers to the resources and equipment to successfully face the pandemic-induced health emergency, as well as the financial means to properly address the socio-economic needs.

In other words, the recent pandemic turned the spotlight on the modern configuration of global economy, based on highly internationally integrated and fragmented production chains which also revealed endogenous structural fragilities. At the same time, the pandemic highlighted that, while being strongly interdependent, economic regions were highly heterogeneous with respect to their resilience in the face of health-related phenomena, their financial strength to respond to economic shocks and their ability to react, from a policy standpoint, to the pandemic itself.

This Forum-The socio-economic consequences of COVID-19: production, employment and welfare, hosted by the Journal of Industrial and Business Economics-gathers 11 contributions that adopt different perspectives in their analyses of the socio-economic impact of the COVID-19 pandemic. A number of key elements take center stage: (i) the structural macroeconomic implications of the pandemic, including investigations of the labour market impact, in a comparative perspective; (ii) the effect of the pandemic on both the Italian and European economy, with particular attention to the risks and opportunities faced by firms and industries highly involved in global production networks; (iii) the impact of COVID-19 on Global Value Chains (GVCs) and the major changes which might occur in the international organization of production, together with a discussion of necessary policy interventions to address the fragilities and increase the resilience of both developed and emerging economies; (iv) the challenges posed by the pandemic to the health sector worldwide, with specific reference to the limits of the current system of intellectual property rights (IPRs) and their implications on the discovery and economic aspects of a vaccine for COVID-19.

Dosi, Fanti and Virgillito in their contribution discuss some of the structural features of the global economy before the pandemic and its potential to further exacerbate them. The authors emphasize the strong inequalities that developed in almost all economies before COVID-19, including China. Inequalities that, differently from what happened with the Plague of the fourteenth century, are not expected to be alleviated by the impact of the virus epidemic. On the contrary, the authors argue that COVID-19 risks to amplify the existing disparities both in access to hospitalization, possibility to work-remotely and benefiting from a stable income, while unevenly increasing the risk of unemployment. In Dosi et al.'s view, the dramatic socio-economic impact of the current pandemic is the combined outcome of, on the one hand, the strong inequalities that had developed in most countries before the spreading of COVID-19; on the other hand, of the consequences of social distancing policies that allowed more stringent social control, which-in turn-risks to exacerbate inequalities by making the distribution of income and power even more polarized than before the pandemic outbreak. 
Highlighting some relevant contradictions affecting the anti-pandemic policy response, Dosi and colleagues make conjectures on possible post-pandemic developments and provide specific warnings as regards the deepening of social and labour market inequalities.

A detailed assessment of the employment implications of the COVID-19 pandemic in Germany, Italy, Poland, Spain, Sweden and the UK is provided by Fana, Torrejón Pérez and Fernandez-Macias. In their contribution they rely on the classification of economic sectors reported in the confinement decrees adopted by Germany, Italy and Spain to estimate and compare the employment impact of each lockdown regime. Their results show a highly heterogeneous picture, both within and between countries. Concerning the 'within' component, Fana and colleagues show that industries most affected by shutdown measures are characterized by the highest share of low-paid jobs and precarious contracts. As for the comparison between countries, they show that Spain, Italy, and the UK, which have been strongly hit by the pandemic, are also those countries that are likely to bear the hardest labour market consequences. In order to explain country-level heterogeneities, Fana et al. focus on industrial specialization patterns and the differences in terms of labour market institutions. The authors therefore argue that the economic crisis induced by the COVID-19 pandemic is likely to accentuate the asymmetries in the labour market conditions across European countries, as well as pre-existing income disparities. To tackle this scenario, they suggest implementing timely and drastic economic interventions at the EU level, with a decisive redistributive component.

The contributions by Celi, Guarascio and Simonazzi; Grabner, Heimberger and Kapeller; and Landesmann, also focus on the challenges posed by the pandemic to the European economy. These articles share a common trait insofar as they highlight, on the one side, the structural asymmetries affecting the European economy before the spreading of COVID-19. On the other side, they investigate the relationship between the structural and political-economic weaknesses of the European integration process and both the magnitude and distribution of the pandemic economic effects. Furthermore, they discuss the possibility for the EU to 'exploit' the COVID19 crisis to fix its 'existential flaws'.

In this regard, Celi, Guarascio and Simonazzi argue that the pandemic-induced economic crisis has (at the time of writing) not triggered sufficient coordinated interventions of mutual protection at the EU level; on the contrary, the response to the COVID-19 crisis by the European authorities seems to open the way to the same mistakes which marked the period following the Great Financial Crisis of 2008. According to their analysis, the centrifugal forces that threaten to disintegrate the European Monetary Union (EMU) can be successfully confronted with a massive and common policy reaction to the financial distress caused by the pandemic. Such a response, however, cannot live up to the task if it fails to remedy the long-term, structural shortcomings that have led to growing disparities among EU's members. In support of their thesis the authors provide a brief review-based on selected key empirical facts - of the institutional and structural causes of the increasing divergence between the EU's core and periphery. In so doing, they shed light on three momentous events: the creation of the monetary union, the 2008 financial crisis and 
the COVID-19 shock. Celi et al. conclude by arguing that European countries are at a crossroad, as they have to choose between allowing the Union to dissolve or reform it radically. They conclude that in the current scenario the only way out for Europe would be to act in a coordinated and collective manner.

Adopting a similar analytical framework, Grabner, Heimberger and Kapeller further discuss the risk of increasing polarization between the production structure of Southern and Northern economies of the Eurozone as a consequence of the pandemic. More precisely, their contribution focuses on the factors through which the pandemic is likely to amplify the uneven vulnerabilities that have been marking the underlying growth models of the core and peripheral EMU countries. Firstly, the authors provide evidence about the diverging tendencies in key economic indicators for both Northern and Southern European countries-the former group comprising Austria, Belgium, Finland, Germany and the Netherlands, while the latter include Greece, Italy, Portugal and Spain, with France occupying an intermediate position. Secondly, they stress the crucial gap in the fiscal policy space the core economies can exploit compared to the one available to peripheral ones. From this analysis follows that the lack of a coordinated policy intervention at European level would accelerate existing divergent dynamics after the lockdown. Furthermore, Grabner et al. discuss to what extent the current European monetary and fiscal policy interventions would be able to address the root causes of structural polarization within the Eurozone. They conclude that recent European policy measures are mostly short-sighted, while long-term interventions would have to foster structural convergence among Eurozone countries_-such as well-targeted industrial policies to manage the reshaping of GVCs and reduce the technological divergences at the regional and national level.

Landesmann in his contribution adopts an analytical framework he recently developed together with Ivano Cardinale (they call it 'structural-political economy', SPE) to study centrifugal and centripetal forces triggered by the economic crises that impacted on the European integration process over the past decade: the financial crisis of 2008 and its aftermath and the COVID-19 crisis. The paper emphasizes the cumulative nature of the impact of these two crises, as they contribute to determine the changing interest and bargaining positions of important actors that define the political dynamics at the EU level. In particular, the author provides an investigation on whether the COVID-19 crisis will lead to a strong collective response and to a qualitative shift in the policy instruments available at the European level, in order to overcome some of the dysfunctionalities of the current set-up of the EU. He analyzes interest positions taken by business, taxpayers and specific social constituencies in the course of these crises which ultimately determines countries' bargaining stances. Landesmann concludes by arguing that cumulativeness, severity and dynamics of the crises can lead to major shifts in interest positions. It follows that both centrifugal as well as centripetal forces are at work and will define the course that European integration will take over the coming years.

The contributions by Strange; Coveri, Cozza, Nascia and Zanfei; and Giammetti, Papi, Teobaldelli and Ticchi, stress the role of both domestic and global value chains as the main transmission channel of the pandemic-induced economic shock on a national and global scale. In particular, the first two articles highlight 
the fragilities of the modern international organization of production, marked by the vertical disintegration of supply chains and the strong involvement of firms and industries in global networks of productions. In doing this, they put emphasis on the strong interconnections of economies and fuel the debate about how the pandemic might reshape the geographical location of value-adding activities and production in GVCs. Coveri et al. also provide a focus on the major economic meltdown of the Italian economy induced by the pandemic. They discuss the vulnerabilities of the country's participation in GVCs and emphasize the role of industrial policies at European level to increase the resilience of strategic industries. Adopting a similar perspective, Giammetti and colleagues deepen the analysis on the Italian production structure. Furthermore, they offer a sophisticated empirical assessment of the impact of lockdown measures on the domestic value chains and provide some guidance for safe and efficient reopening policies.

In his contribution, Strange focuses on the effect of the pandemic on firms' strategies, with special attention to their involvement in GVCs. The paper provides a careful evaluation of the pros and cons of the geographical dispersion as opposed to domestic concentration of value chain activities by firms, thus addressing the location strategies of Multinational Corporations (MNCs) and how they might change once pandemic restrictions are lifted. In particular, Strange assesses the merits of international diversification of suppliers against reshoring strategies, as well as the potential impact of large-scale events like pandemics on the governance of GVCs. After suggesting corporate policies aimed at increasing the resilience of the supply chains in order to minimize future disruptions in key productions, Strange concludes by arguing that reshoring strategies and a shortening of supply chains would entail more costs than benefits in terms of both MNCs' economic performance and overall well-being. It follows that, according to the author, a wider international diversification and greater externalization of production is likely to be the best response to future pandemic events.

The arguments supported by Strange are at least partially contradicted by the contribution of Coveri, Cozza, Nascia and Zanfei. The authors firstly provide evidence about the economic impact of the pandemic on both the European and Italian economy. Secondly, they shed light on the growing integration of the Italian economy in global networks of production, with specific reference to backward linkages with China as well as the rest of the world. More precisely, they show that the overall backward linkages in GVCs of the Italian economy with China have been increasing in the last two decades and are roughly equal to the ones registered by the US in 2015. However, Italy's integration in European and North American GVCs - heavily affected by the pandemic as well-is still considerably higher. In what follows, Coveri et al. take the automotive sector as an example to illustrate the shifting specialization pattern of Italy in GVCs, from the integrated production of cars for the final markets to a more upstream position, i.e. as global supplier of components for the car industry. Against the background of this empirical analysis, the authors discuss the limits of an extreme specialization induced by the modern international fragmentation of production. In addition, they offer several arguments in favor of a well-targeted European industrial 
policy plan aimed at the consolidation of resilient GVCs on a continental scale to expand the production matrix of firms and pursue common development goals.

Giammetti, Papi, Teobaldelli and Ticchi analyze the economic consequences of lockdown measures on the Italian economy relying on both network and Input-Output analytical tools. More specifically, the authors investigate the structure of Italy's domestic value chains, taking into account the interlinkages across sectors and their role in the transmission of the economic shock triggered by the pandemic restrictions. In this way, they are able to identify those 'systemically important' industries which are central to the production networks and whose shutdown leads to major disruptions in national production through both direct and indirect propagation mechanisms. Based on this analysis, Giammetti et al. estimate that the lockdown imposed by the Italian Government on such key sectors would have reduced GDP by about $52 \%$. This contribution therefore provides a potentially useful guidance to the design of reopening policy plans, suggesting to favor those industries which entail higher economic benefits and fewer risks for workers. Nonetheless, based on both the value-generating capacity and the occupational structures of industries, the authors stress that policy interventions aimed at minimizing value added losses should be implemented in conjunction with redistributive fiscal policies to support the most vulnerable population groups.

The last three contributions included in this Forum are not less relevant for the discussion on the socio-economic impact of COVID-19, as they touch on topics such as the policy implications for the survival and evolution of Small- and Mediumsized Enterprises (SMEs) in both the short- and long-term (Juergensen, Guimón and Narula); the limits set by the patent system in the pharmaceutical industry on the discovery and especially on the widespread economic affordability of a vaccine for COVID-19 (Marengo); and the impact of the pandemic on the Indian economy, with special attention to the (flawed) policy response promoted by the Indian Government (Ghosh).

The contribution by Juergensen, Guimón and Narula outlines the paramount role of SMEs in the European economy. They give an account of the heterogenous composition of SMEs in the manufacturing sector to describe the differentiated impact that the pandemic has had on them and suggest adopting policy interventions accordingly. In particular, the authors take advantage of a distinction of SMEs in three main types-i.e. stand-alone, specialized supplier and knowledge-based SMEs. Furthermore, they distinguish between immediate effects of the lockdown regime across Europe for the survival of SMEs, and the longer-term implications regarding their recovery and growth. They argue that-differently from what happened after the outbreak of the financial crisis of 2008-European policy makers have demonstrated to be fully aware of the key role played by manufacturing SMEs and acted accordingly through promoting specific wage support schemes and offering several forms of financial support to alleviate their liquidity constraints during the pandemic. Most importantly, Juergensen et al. stress that, as the lockdown measures will be progressively eased, the focus on the survival of SMEs should give way to more structural and longer-term policy interventions. In this context, they argue that both European and national economic policy authorities should promote "transformative" interventions, focused on innovation, internationalization and 
networking, with the aim of shaping the evolution of SMEs rather than fixing shortterm market failures.

A careful examination of the flaws highlighted by COVID-19 of the current IPR regime in the pharmaceutical sector is at the center of Marengo's contribution. The author first provides a brief historical reconstruction of the birth of patents, then focuses on the merits as well as social inefficiencies of the market-based functioning of the patent system in the pharmaceutical sector. In particular, Marengo illustrates the reasons why monopolies in the pharmaceutical market are likely to produce huge social costs, while the protection afforded to pharmaceutical companies by current IPRs has not stimulated the industry's rate of innovativeness. Moreover, the author argues that the patent system does not provide the right incentives for pharmaceutical companies to develop vaccines-including the one to combat COVID-19-so that the role of public sector for both the introduction and accessibility to the entire population of this medicine is crucial.

Finally, the contribution by Ghosh investigates the socio-economic impact of COVID-19 in India and outlines the shortcomings related to the central government's response to the pandemic. More precisely, the author claims that the design of the lockdown measures promoted by the national government have created extreme economic hardship for the Indian population, especially for the over 100 million migrant workers. Furthermore, the restrictions have not been accompanied by interventions to support effective demand and alleviate the social burden due to the lack of livelihoods, in a country where around 95\% of workers are informal. Nonetheless, this "centralization without coordination" of policy interventions by the central government has led to the ineffectiveness of production shutdowns in reducing the spread of COVID-19, while inducing a generalized collapse of the economy. Ghosh concludes that a successful and socially inclusive strategy to combat the pandemic would have required a different macroeconomic strategy, featured by a massive expansion of the Indian public health system.

Publisher's Note Springer Nature remains neutral with regard to jurisdictional claims in published maps and institutional affiliations. 